\title{
Beclin-1 as a neutrophil-specific immune checkpoint
}

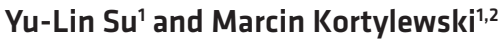 \\ 1Department of Immuno-Oncology and ²Center for Cene Therapy, Beckman Research Institute at City of Hope Comprehensive Cancer Center, Duarte, California, USA.
}

\begin{abstract}
Neutrophils are early wound healing and inflammation regulators that, due to functional plasticity, can adopt either pro- or antitumor functions. Until recently, beclin-1 was a protein known mainly for its role as a critical regulator of autophagy. In this issue of the $J C I$, Tan et al. describe the effects of the beclin-1 conditional myeloid cell-specific deletion in mice, in which immunostimulation resulted in hypersensitive neutrophils. The chronic proinflammatory effect of these neutrophils triggered spontaneous B cell malignancies to develop. Such tumorigenic effects were mediated primarily by IL-21 and CD40 signaling, leading to the upregulation of tolerogenic molecules, such as IL-10 and PD-L1. The authors went on to examine samples derived from patient lymphoid malignancies and showed that beclin-1 expression in neutrophils positively correlated with pre-B cell leukemia/lymphoma. Overall, the study provides an elegant model for neutrophil-driven carcinogenesis and identifies potential targets for immunotherapy of B cell malignancies.
\end{abstract}

\section{Neutrophils in the tumor microenvironment}

Tumor progression is defined by both cancer cell-intrinsic effects, related to genetic and epigenetic phenomena, and extrinsic influences of various nonmalignant stromal cells (1). In particular, macrophages and myeloid-derived suppressor cells (MDSCs) have attracted the attention of scientists and clinicians for their profound role in supporting tumor progression, angiogenesis, and immune evasion and thus their impressive therapeutic potential (2-4). Until recently, less was known about neutrophils, which often comprise the most abundant subset of myeloid cells and are more challenging to study due to their phenotypic diversity and limited life span.

Neutrophils are important early regulators of wound healing and chronic inflammation. While they were initially overshadowed by tumor-associated macrophages, strong evidence suggests that neutrophils infiltrate the tumors and actively participate in tumor growth and metastasis (5).
However, due to their functional plasticity, neutrophils can have either tumorinhibitory (N1 type) or tumor-promoting (N2 type) effects. The immature N2 neutrophils share surface markers and morphology with polymorphonuclear MDSCs (PMN-MDSCs), which are also immature neutrophils that have immunosuppressive potential $(3,5)$. GM-CSF-induced activation of STAT3 transcription factor is the key molecular mechanism that drives neutrophil expansion and differentiation. Tumor-derived factors, such as IL-6, stem cell factor (SCF), or GM-CSF, often hijack this mechanism to accumulate immature neutrophils. Importantly, the chronic STAT3 activity in tumor-associated neutrophils (TANs) hampers their cytotoxic activity and can induce proangiogenic and immunosuppressive phenotypes $(6,7)$.

\section{Beclin-1 as a key regulator of neutrophil activity}

The study by Tan and colleagues in the current issue of the JCI brings into light a new

\section{Related Article: p. 5261}

Conflict of interest: The authors have declared that no conflict of interest exists.

molecular mechanism regulating proinflammatory and tumorigenic properties of neutrophils, distinct from the PMN-MDSC phenotype $(8,9)$. The authors focused on the role of beclin-1, an important positive regulator of early autophagy stages and a potential tumor suppressor (10). In fact, the heterozygous deletion of beclin-1 gene (Becn1) resulted in spontaneous formation of lymphomas and lung and liver cancers in mice (11). While the cytoprotective and immunogenic effects of autophagy in cancer cells have been intensely studied, the autophagy process proved to be highly complex, with conflicting effects and poor druggability. Interestingly, a previous study from the same research group indicated that, beyond the role in autophagy, beclin-1 also controls production of type I IFN and thereby controls innate immunity (12). Together, these observations suggested that beclin-1 might, at least partly, act as a cancer cell-autonomous tumor suppressor through activity in nonmalignant, innate immune cells.

Tan et al. found that the myeloid-specific deletion of Becn1 (Becn1 $\left.1^{\backslash M}\right)$ resulted in hypertrophy of lymphoid organs, such as spleen and lymph nodes, in mice. Selective neutrophil and B cell expansion, without changes among monocyte or macrophage populations, caused these histopathological aberrations. Specifically, LPS stimulated TLR4 on neutrophils to produce proinflammatory mediators, including TNF- $\alpha$, ROS, CD4OL, or cytokines such as IL-1 $\beta$, IL-17, and IL-21. Due to high inflammatory-factor levels, Becn $1^{\Delta M}$ mice developed an increased sensitivity to endotoxin shock. A series of careful in vitro studies suggested that beclin-1 interacts with MEKK3 kinase upstream from p38 signaling, which plays a key role in regulating proinflammatory mediators. Using Becn $1^{\Delta M}$ mouse models with targeted ablation of MEKK3 or p38 encoding genes, the authors verified that the upregulation of proinflammatory cytokines in Becn $1^{4 M}$ neutrophils depends on the MEKK3/p38 signaling pathway. Furthermore, they found that beclin-1 was directly responsi- 


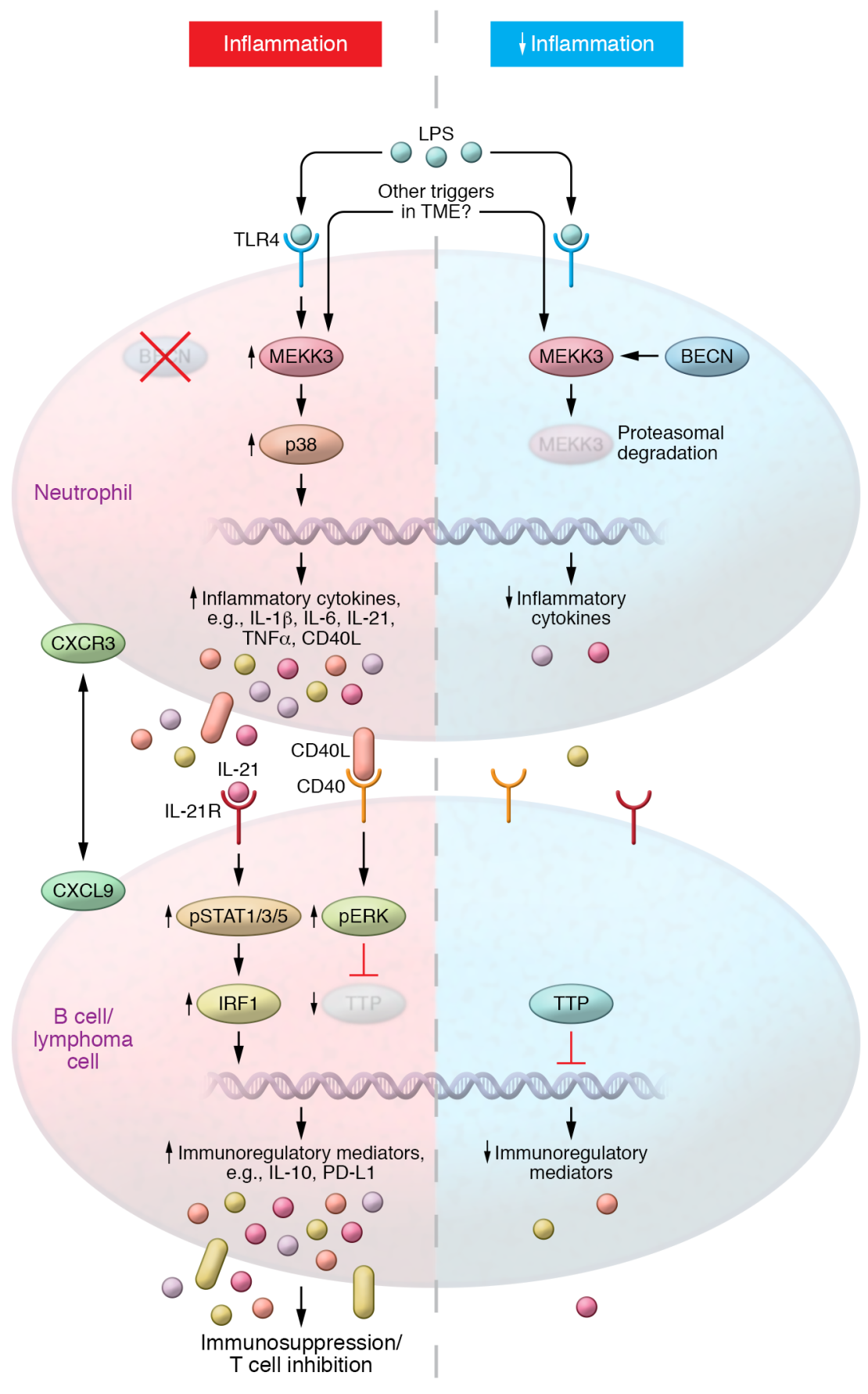

ble for the ubiquitination and proteasomal degradation of MEKK3, thereby mitigating downstream inflammatory signaling in wild-type neutrophils (Figure 1 and ref. 9).

\section{Crosstalk between Becn1-} deficient neutrophils and $B$ cells Intriguingly, about $20 \%-40 \%$ of Becn $1^{\Delta M}$ mice developed spontaneous and highly proliferative lymphomas with a pre-B lymphoblastic lymphoma phenotype. Given that Becn1 deletion was limited to myeloid cells and not B cells, these results
Figure 1. Beclin-1 operates as an immune checkpoint regulator in neutrophils. A model for the interaction between Becn1-deficient neutrophils and B cells or B cell leukemia/lymphoma cells in vivo shows beclin-1 deficiency unleashes inflammatory TLR signaling in neutrophils by stabilizing MEKK3, leading in turn to excessive $\mathrm{p} 38$ signaling. As a result, hyperactive neutrophils initiate CXCR3/CXCL9-mediated B cell chemotaxis. The release of proinflammatory mediators, such as IL-21, and the expression of CD40 ligand by neutrophils triggers MAPK and Jak/STAT signaling in B cells, resulting in the production of immunosuppressive regulators IL-10 and PD-L1. These tolerogenic effects likely contribute to the development of B cell ALL.

identified a population of tumor-promoting neutrophils with so-called B cell helper phenotype assisting in the transition from autoimmune manifestations to chronic lymphocytic leukemia or lymphoma $(13,14)$.

Tan et al. next investigated the molecular mechanisms driving B cell lymphomagenesis in Becn1 $1^{\Delta M}$ mice. The interaction of B cells with Becn1-deficient neutrophils resulted in increased activity of STATs (STAT1, STAT3, and STAT5) as well as ERK and JNK kinases downstream from IL-21 and/or CD40 receptors. The B cells from Becn1 $1^{\Delta M}$ mice upregulated expression of several STAT1/3 targets, such as $C d 274$ (PD-L1), Cxcl9, Irf1, Socs1 or Socs3, and B cell activation markers (Il1O, Saa3) (15). Also upregulated was a gene encoding a key cytosolic DNA sensor (Mb21d1), better known as cyclic GMP-AMP synthase (cGAS), which shows dichotomous pro- and antitumor effects (16). Both Jak/STAT3 and ERK signaling contributed to PD-L1 expression through direct promoter activation and potential transcript stabilization (9).

Tovalidate the therapeutic implications of these findings, Tan et al. used antibodies to neutralize PD-L1 or IL-21R in lymphoma-bearing Becn $1^{\Delta M}$ mice. The elimination of IL-21 as well as PD-1 immune checkpoint blockade triggered tumor infiltration by cytotoxic CD ${ }^{+} \mathrm{T}$ cells, thus supporting the role of both molecules in the neutrophil and B cell crosstalk (9).

Finally, Tan et al. provided enticing evidence for beclin-1 as an immune checkpoint regulator in human B cell malignancies, such as pre-B acute lymphoblastic leukemia/lymphoma (ALL). The neutrophils in tested pre-B cell ALL patients showed lower on average expression of beclin-1, 
with increased neutrophil activation as measured by formation of neutrophil extracellular traps (NETs). Correspondingly, the gene expression analysis using publicly available databases confirmed the correlation between the expression of neutrophil marker (LY6G) and upregulation of both IL-21 and PD-L1 in a large group of recurrent pre-B cell ALL patients (9).

\section{Remaining questions and therapeutic implications}

The report by Tan et al. sheds light on the new role of beclin-1 as a potential neutrophil-specific immune-checkpoint molecule operating in inflammation and cancer (9). At the same time, the study raises a number of questions warranting further investigation. Is beclin-1 dynamically regulated in neutrophils by inflammatory mediators, as suggested by studies demonstrating the role of $\mathrm{NF}-\kappa \mathrm{B}$ and various miRNAs in Becn1 expression (17)? Would STAT3 counteract these effects in TANs and repress Becn1, as shown before in cancer cells (18)? Which innate immune receptors in neutrophils (TLRs, cGAS/STING, RIG-I) are controlled by beclin-1 to prevent inflammatory and tumorigenic effects? More in-depth studies should evaluate further the contribution of neutrophils and beclin-1-controlled IL-21 production in the pathogenesis of human B-ALL. Due to direct cytotoxic effects on malignant $\mathrm{B}$ cells, IL-21 is being broadly explored for therapy of B cell lymphomas, albeit with limited success as a monotherapy (19). The current study indicates that IL-21 can have an opposite, tumor-promoting effect in mice, thus underscoring the importance of testing immune mediators in the broader context of the immune cell network. The neutralization of IL-21 alone or together with PD-L1 could provide new immunotherapeutic approaches to recurrent adult pre-B cell ALL, since the emerging anti- body and CAR T cell immunotherapies are often hampered by the emergence of CD19- or CD20-negative cancer clones (20). Interestingly, the correlation of high neutrophil infiltration and poor patient outcomes have been previously reported in brain, breast, or lung cancers (21), which are also known for reduced beclin-1 levels (17). Thus, it is tempting to suggest that the role of beclin-1 as a neutrophil-specific checkpoint and tumor suppressor could reach beyond $\mathrm{B}$ cell malignancies.

\section{Acknowledgments}

This work was supported by the National Cancer Institute of the NIH under grant numbers R01CA213131, P50CA107399, and R01CA215183, and by the Department of Defense (Prostate Cancer Research Program), grant number W81XWH-16-1-0499 (to $\mathrm{MK})$. The content is solely the responsibility of the authors and does not necessarily represent the official views of the NIH.

Address correspondence to: Marcin Kortylewski, Beckman Research Institute at City of Hope Comprehensive Cancer Center, BCK 3111, 1500 East Duarte Road, Duarte, California 91010, USA. Phone: 626.218. 4120; Email: mkortylewski@coh.org.

1. Hanahan D, Weinberg RA. Hallmarks of cancer: the next generation. Cell. 2011;144(5):646-674.

2. DeNardo DG, Ruffell B. Macrophages as regulators of tumour immunity and immunotherapy. Nat Rev Immunol. 2019;19(6):369-382.

3. Kumar V, Patel S, Tcyganov E, Gabrilovich DI. The nature of myeloid-derived suppressor cells in the tumor microenvironment. Trends Immunol. 2016;37(3):208-220.

4. Kortylewski M, Moreira D. Myeloid cells as a target for oligonucleotide therapeutics: turning obstacles into opportunities. Cancer Immunol Immunother. 2017;66(8):979-988.

5. Coffelt SB, Wellenstein MD, de Visser KE. Neutrophils in cancer: neutral no more. Nat Rev Cancer. 2016;16(7):431-446.

6. Hillmer EJ, Zhang H, Li HS, Watowich SS. STAT3 signaling in immunity. Cytokine Growth Factor
Rev. 2016;31:1-15.

7. Kujawski M, Kortylewski M, Lee H, Herrmann A, Kay H, Yu H. Stat 3 mediates myeloid cell-dependent tumor angiogenesis in mice. JClin Invest. 2008;118(10):3367-3377.

8. Moses K, Brandau S. Human neutrophils: Their role in cancer and relation to myeloidderived suppressor cells. Semin Immunol. 2016;28(2):187-196

9. Tan P, et al. Myeloid loss of Beclin 1 promotes $\mathrm{PD}-\mathrm{L} 1{ }^{\text {hi }}$ precursor B cell lymphoma development. J Clin Invest. 2019;129(12):5261-5277.

10. Dikic I, Elazar Z. Mechanism and medical implications of mammalian autophagy. Nat Rev Mol Cell Biol. 2018;19(6):349-364.

11. Qu X, et al. Promotion of tumorigenesis by heterozygous disruption of the beclin 1 autophagy gene. J Clin Invest. 2003;112(12):1809-1820.

12. Jin S, et al. USP19 modulates autophagy and antiviral immune responses by deubiquitinating Beclin-1. EMBO J. 2016;35(8):866-880.

13. Puga I, et al. B cell-helper neutrophils stimulate the diversification and production of immunoglobulin in the marginal zone of the spleen. Nat Immunol. 2011;13(2):170-180.

14. Sangaletti S, et al. Defective stromal remodeling and neutrophil extracellular traps in lymphoid tissues favor the transition from autoimmunity to lymphoma. Cancer Discov. 2014;4(1):110-129.

15. Huynh J, Chand A, Gough D, Ernst M. Therapeutically exploiting STAT3 activity in cancer - using tissue repair as a road map. Nat Rev Cancer. 2019;19(2):82-96

16. Ng KW, Marshall EA, Bell JC, Lam WL. cGASSTING and cancer: dichotomous roles in tumor immunity and development. Trends Immunol. 2018;39(1):44-54.

17. Wechman SL, et al. New insights into Beclin-1: evolution and pan-malignancy inhibitor activity. Adv Cancer Res. 2018;137:77-114.

18. Miao LJ, Huang FX, Sun ZT, Zhang RX, Huang SF, Wang J. Stat3 inhibits Beclin 1 expression through recruitment of HDAC3 in nonsmall cell lung cancer cells. Tumour Biol. 2014;35(7):7097-7103.

19. Bhatt S, Sarosiek KA, Lossos IS. Interleukin 21 its potential role in the therapy of B-cell lymphomas. Leuk Lymphoma . 2017;58(1):17-29.

20. Terwilliger T, Abdul-Hay M. Acute lymphoblastic leukemia: a comprehensive review and 2017 update. Blood Cancer J. 2017;7(6):e577.

21. Gentles AJ, et al. The prognostic landscape of genes and infiltrating immune cells across human cancers. Nat Med. 2015;21(8):938-945. 